\title{
PROJECTIVE MODULES OVER SUBRINGS OF $k[X, Y]$
}

\author{
BY
}

DAVID F. ANDERSON

\begin{abstract}
In this paper we study projective modules over subrings of $k[X, Y]$. Conditions are given for projective modules to decompose into free $\oplus$ rank 1 modules. Our main result is that if $k$ is an algebraically closed field and $A$ a subring of $B=k[X, Y]$ with $A \subset B$ integral and $\operatorname{sing}(A)$ finite, then all f.g. projective $A$-modules have the form free $\oplus$ rank l. We also give several examples of subrings of $k[X, Y]$ which have indecomposable projective modules of rank 2.
\end{abstract}

1. Introduction. Seshadri [14] first showed that all f.g. projective $k[X, Y]-$ modules are free. It is well known that if $A$ is an arbitrary subring of $k[X, Y]$, then not all f.g. projective $A$-modules are free. For example, when $A=$ $k\left[X^{2}, X^{3}, Y\right], \operatorname{Pic}(A)=k[Y]$. When $A$ is an affine subring of $k[X, Y]$, f.g. projective $A$-modules have the form free $\oplus$ rank 2 by Serre's Theorem [3, p. 173]. In this paper we study cases where this decomposition can be improved to free $\oplus$ rank 1. In [1] or [2] the author proved the following theorem.

THEOREM 1.1. Let $A$ be an affine subring of $k[X, Y]$ generated by monomials; then:

(1) All f.g.projective A-modules stably have the form free $\oplus$ rank 1 .

(2) If $A$ is normal, then all f.g. projective $A$-modules are free.

In $\$ 2$ we establish notation and a few preliminary results.

In $\$ 3$ we study conditions for $\mathrm{f}$. g. projective $A$-modules to decompose into free $\oplus$ rank 1 modules when $A$ has $\operatorname{dim} 2$. Presumably these results are well known; compare [9].

Our main theorem (4.1) is proved in $\S 4$. We show that if $k$ is an algebraically closed field and $A$ an affine subring of $B=k[X, Y]$ with $\operatorname{sing}(A)$ finite and $A \subset B$ integral, then all f.g. projective $A$-modules have the form free $\oplus$ rank 1. A special application of this theorem is to rings of invariants. This is discussed in $\$ 5$.

In $\$ 6$ we give several examples of subrings $A$ of $k[X, Y]$ which have indecomposable projective $A$-modules of rank 2 . These examples show that the projective $A$-module structure depends on the field $k$.

This paper constitutes part of the author's dissertation at the University of

Presented to the Society, January 29, 1977; received by the editors December 15, 1976.

AMS (MOS) subject classifications (1970). Primary 13C05, 13C10, 13D15, 18F25, $18 \mathrm{G} 05$.

- American Mathematical Society 1978 
Chicago. The author would like to thank his advisor, Professor M. Pavaman Murthy, for his many helpful suggestions.

2. Preliminaries. All rings will be commutative with $1 . A$ will denote a ring, $M$ a maximal ideal of $A$, and $k$ a field. Our $K$-theory notation follows [3], while $K_{2}$ will be Milnor's $K_{2}$ [6]. $\operatorname{spec}(A)$ will denote the set of all prime ideals of $A$ with the Zariski topology. For $I$ an ideal of $A, V(I)=\{P \in \operatorname{spec}(A) \mid P$ $\supset I\} \cdot \max (A)$ is the subset of $\operatorname{spec}(A)$ consisting of maximal ideals. The dimension of a subset of $\operatorname{spec}(A)$ will be the usual combinatorial dimension. The dimension of a ring $A$ will always mean Krull dimension and will be denoted by $\operatorname{dim} A$. We will denote the homological dimension of an $A$ module $N$ by $\operatorname{~hd}_{A} N$. If $N$ is an $A$-module, then supp $N=\left\{P \in \operatorname{spec}(A) \mid N_{P}\right.$ $\neq 0\}$. The group of units of a ring $A$ will be denoted by $A^{*}$. We will abbreviate short exact sequence by SES. The set of singularities of $A$ will be denoted by $\operatorname{sing}(A)=\left\{P \in \operatorname{spec}(A) \mid A_{P}\right.$ is not regular $\}$.

We recall that $\tilde{K}_{0}(A)$ is the subgroup of $K_{0}(A)$ generated by elements of the form $[P]-\left[A^{\mathrm{rank} P}\right]$, where $P$ is an f.g. projective $A$-module. There is the natural determinant homomorphism det: $K_{0}(A) \rightarrow \operatorname{Pic}(A) \operatorname{defined}$ by $\operatorname{det}([P])$ $=\Lambda^{n}(P)$ where $n=\operatorname{rank} P$ and $\operatorname{Pic}(A)$ is the group of isomorphism classes of f.g. projective $A$-modules of rank 1 . Clearly det induces an epimorphism det: $\tilde{K}_{0}(A) \rightarrow \operatorname{Pic}(A)$; the kernel of this map will be denoted by $S K_{0}(A)$.

The following elementary lemma will be stated without proof.

LEMMA 2.1. The following are equivalent.

(1) det is an isomorphism.

(2) $S K_{0}(A)=0$.

(3) All f.g. projective A-modules of constant rank stably have the form free $\oplus$ rank 1 .

(4) Let $P$ be a f.g. projective $A$-module of rank $n$; then $\Lambda^{n}(P) \approx A$ iff $P$ is stably free.

A commutative square of rings

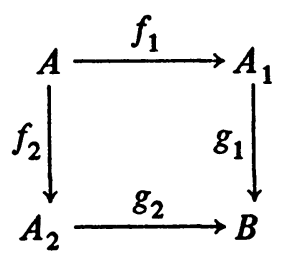

is said to be cartesian if given $x \in A_{1}$ and $y \in A_{2}$ such that $g_{1}(x)=g_{2}(y)$, then there exists a unique $z \in A$ such that $f_{1}(z)=x$ and $f_{2}(z)=y$.

THEOREM 2.2 [3, p. 481]. Given a cartesian square of rings with $g_{1}$ surjective, wa have the following "Mayer-Vietoris" exact sequences 


$$
\begin{aligned}
& 0 \rightarrow A^{*} \rightarrow A_{1}^{*} \oplus A_{2}^{*} \rightarrow B^{*} \stackrel{\partial}{\rightarrow} \operatorname{Pic}(A) \rightarrow \operatorname{Pic}\left(A_{1}\right) \oplus \operatorname{Pic}\left(A_{2}\right) \rightarrow \operatorname{Pic}(B), \\
& K_{1}(A) \rightarrow K_{1}\left(A_{1}\right) \oplus K_{1}\left(A_{2}\right) \rightarrow K_{1}(B) \stackrel{\partial}{\rightarrow} \tilde{K}_{0}(A) \\
& \rightarrow \tilde{K}_{0}\left(A_{1}\right) \oplus \tilde{K}_{0}\left(A_{2}\right) \rightarrow \tilde{K}_{0}(B) .
\end{aligned}
$$

Using the natural determinant maps we can connect sequences (1) and (2) to obtain the following commutative diagram with exact rows and columns.

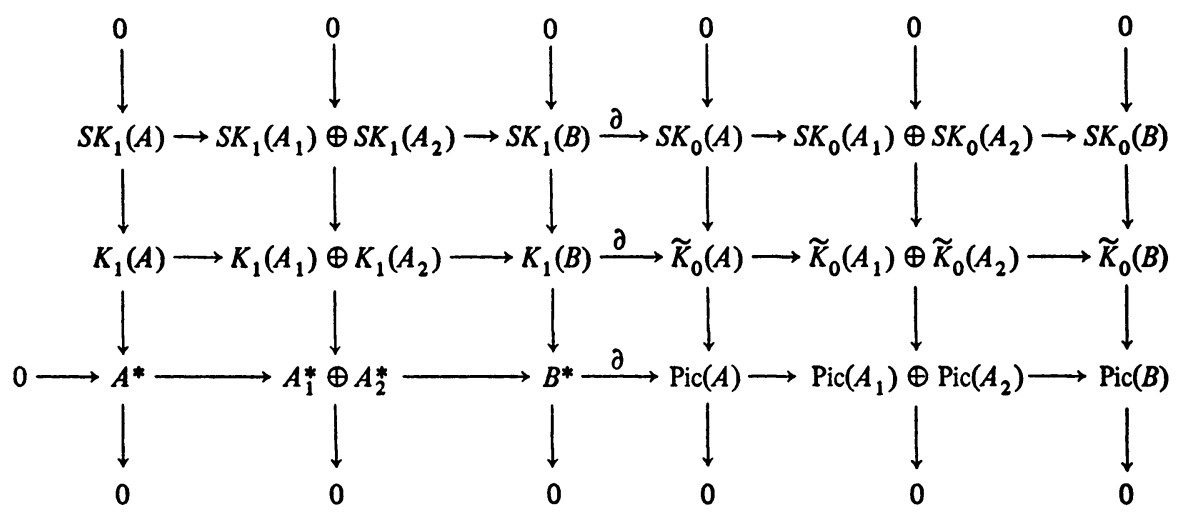

We have the following immediate lemma.

LEMMA 2.3. Suppose that $S K_{0}\left(A_{1}\right)=S K_{0}\left(A_{2}\right)=0$; then

(1) $S K_{0}(A)=\partial\left(S K_{1}(B)\right)$.

(2) If $S K_{1}(B)=0$, then $S K_{0}(A)=0$.

(3) If $h$ is an isomorphism, then $S K_{1}(B) \approx S K_{0}(A)$.

$S K_{0}(A)=0$ just implies that stably all f.g. projective $A$-modules have the form free $\oplus$ rank 1 . To remove this stability requirement we will often use the following cancellation theorem.

TheOREM 2.4 (MURTHY-SwAN [9]). Let $A$ be an affine ring of $\operatorname{dim} \leqslant 2$ over an algebraically closed field. If $P$ is a f.g. projective $A$-module and $A \oplus P \approx A$ $\oplus Q$, then $P \approx Q$.

$\left(a_{0}, \ldots, a_{n}\right) \in A^{n+1}$ is unimodular if $A a_{0}+\cdots+A a_{n}=A$. The stable range of $A$, denoted by $\operatorname{sr}(A)$, is $<d$ if given any unimodular row $\left(a_{0}, \ldots, a_{d}\right)$, there exist $c_{0}, \ldots, c_{d-1} \in A$ so that $\left(a_{0}+c_{0} a_{d}, \ldots, a_{d-1}+\right.$ $\left.c_{d-1} a_{d}\right) \in A^{d}$ is still unimodular. For $N$ an $A$-module, $x \in N$ is unimodular if $N=A x \oplus N^{\prime}$.

3. Rings of $\operatorname{dim} 2$. If $A$ is an affine domain, $\operatorname{sing}(A)$ is closed [5, p. 245]. However, $\operatorname{sing}(A)$ need not be closed in general [10]. If $A$ is an affine domain of $\operatorname{dim} 2$, then $\operatorname{dim} \operatorname{sing}(A)<1$ because $0 \notin \operatorname{sing}(A)$. If, in addition, $A$ is 
normal, then $\operatorname{sing}(A)$ has $\operatorname{dim} 0$ and, hence, is finite because $A_{P}$ is a DVR for all ht 1 prime ideals $P$.

We note that for $M \in \max (A), M \notin \operatorname{sing}(A)$ iff $A_{M}$ is regular iff hd $A<$ $\infty$. If $M \in \max (A)$, then $\operatorname{Ext}_{A}^{1}(M, A) \approx \operatorname{Ext}_{A}^{2}(A / M, A)$ is annihilated by $M$. Thus $\operatorname{Ext}_{A}^{1}(M, A) \approx \operatorname{Ext}_{A_{M}}^{1}\left(M_{M}, A_{M}\right)$. If $M \notin \operatorname{sing}(A)$ and $\operatorname{dim} A_{M}=2$, then $A_{M}$ is a regular local ring of $\operatorname{dim} 2$, so $\operatorname{Ext}_{A}^{1}(M, A) \approx A / M$.

LEMMA 3.1 (SERRE [13]). Let $A$ be a noetherian ring and $M$ an f.g. A-module with $\operatorname{hd}_{A} M<1$. If $\operatorname{Ext}_{A}^{1}(M, A)$ is generated by $r$ elements, then there is a SES $0 \rightarrow A^{r} \rightarrow P \rightarrow M \rightarrow 0$ where $P$ is a f.g. projective $A$-module.

LEMMA 3.2 [9]. Let $M$ be a maximal ideal of $A$ such that $A_{M}$ is a regular local ring of $\operatorname{dim} 2$. If there is a SES $0 \rightarrow Q \rightarrow P \rightarrow M \rightarrow 0$ where $P$ is a f.g. projective $A$-module of rank 2 , then $\Lambda^{2}(P) \approx Q$.

CoROllary 3.3. Let $I$ be an ideal of $A$ of ht 2 with $V(I)$ finite and for all $M \in V(I), A_{M}$ is a regular local ring of $\operatorname{dim} 2$. Then for any $S E S 0 \rightarrow Q \rightarrow P$ $\rightarrow I \rightarrow 0$, where $P$ is a f.g. projective $A$-module of rank $2, \Lambda^{2}(P) \approx Q$.

LEMMA 3.4. Let $A$ be a commutative noetherian ring and $M$ a maximal ideal such that $A_{M}$ is a regular local ring of $\operatorname{dim} 2$; then $[A / M] \in S K_{0}(A)$.

Proof. Here we consider $K_{0}(A)$ as $K_{0}(H(A))$, where $H(A)$ is the category of f.g. $A$-modules with finite homological dimension [3, p. 407]. By Lemma 3.1 there is a SES $0 \rightarrow A \rightarrow P \rightarrow M \rightarrow 0$ where $\mathrm{P}$ is a f.g. projective $A$-module of rank 2. By Lemma 3.2, $\Lambda^{2}(P) \approx A$. So

$$
[A / M]=[A]-[M]=[A]-[P]+[A]=\left[A^{2}\right]-[P]
$$

but $\Lambda^{2}(P) \approx A$, so $\left[A^{2}\right]-[P] \in S K_{0}(A)$.

We note that if $A_{M}$ is a regular local ring of $\operatorname{dim} 1$, it need not be true that $[A / M] \in S K_{0}(A)$. For in this case $M$ is actually projective, so $[A / M] \in$ $S K_{0}(A)$ iff $\Lambda^{1}(M)=M \approx A$, that is, $M$ is principal. We next show that in many cases the $[A / M]$ 's generate $S K_{0}(A)$.

Proposition 3.5. Let $A$ be a commutative noetherian ring of $\operatorname{dim} 2$ and $X=\{M \in \max (A) \mid$ ht $M<1\}$. Assume that $Y=X \cup \operatorname{sing}(A) \subset Z$, where $Z \subset \operatorname{spec}(A)$ is a closed set of $\operatorname{dim}<1$. Then $S K_{0}(A)$ is generated by $[A / M]$ for $M \in \max (A) \backslash Y$.

Proof. Any element of $S K_{0}(A)$ has the form $[P]-\left[A^{2}\right]$, where $P$ is a f.g. projective $A$-module of $\operatorname{rank} 2$ with $\Lambda^{2}(P) \approx A$. It is sufficient to find $f \in P^{*}=\operatorname{Hom}_{A}(P, A)$ such that $A / \operatorname{im} f$ is $\operatorname{artinian}$ and $\operatorname{supp}(A / \operatorname{im} f) \cap Z$ $=\varnothing$.

For let $I=\operatorname{im} f$ and $V(I)=\left\{M_{1}, \ldots, M_{n}\right\} \subset \max (A) . \operatorname{supp}(A / I) \cap Z$ $=\varnothing$, so each $\left[A / M_{i}\right] \in S K_{0}(A)$ by Lemma 3.4. We have a SES $0 \rightarrow Q \rightarrow P$ 
$\stackrel{f}{\rightarrow} I \rightarrow 0$ where $Q=\operatorname{ker} f$. Since each $M_{i} \notin \operatorname{sing}(A)$, hd $A_{M_{i}} I_{M_{i}}<1$ and thus $\mathrm{hd}_{A} I<1$. So hd $A=0$ and $Q$ is a f.g. projective $A$-module. By Corollary 3.3, $Q \approx \Lambda^{2}(P) \approx A$. Thus $[P]=[A]+[I]$ in $K_{0}(A)$.

$A / I$ is artinian and thus has a composition series $A / I=N_{0} \supset N_{1}$ $\supset \cdots \supset N_{l}=0$, where each $N_{i} / N_{i+1} \approx A / M_{j}$ for some $M_{j} \in V(I)$. So in $K_{0}(A),[A / I]=\Sigma\left[N_{i} / N_{i+1}\right]=\Sigma\left[A / M_{j}\right]$, and thus

$$
[P]-\left[A^{2}\right]=[A]+[I]-\left[A^{2}\right]=-[A / I]=-\sum\left[A / M_{j}\right] \text {. }
$$

So $S K_{0}(A)$ is generated by $\{[A / M] \mid M \in \max A \backslash Z\}$.

Now we show how to find the desired $f \in P^{*}$. Let $Q=P^{*}$, then $Q$ is also a f.g. projective $A$-module of rank 2. $Z$ is closed, so $Z=V(J)$ with $\operatorname{dim} A / J<$ 1. By Serre's Theorem [3, p. 173], there is an $x \in Q$ so that $\bar{x}$ is unimodular in $\bar{Q}=Q / J Q$ and $O_{Q}(x)+J=A$ where $O_{Q}(x)=\left\{g(x) \mid g \in Q^{*}\right\}$. Thus there is an $a \in J$ with $O_{Q}(x)+A a=A$. Then $(a, x)$ is unimodular in $A \oplus Q$. By [15, p. 13], there exists a $y \in Q$ so that ht $O_{Q}(x+a y) \geqslant 2$. Let $f=x+a y \in$ $Q=P^{*}$, then $A / \operatorname{im} f$ is artinian and $O_{Q}(f)+J=A$, which implies $\operatorname{supp}(A / \operatorname{im} f) \cap Z=\varnothing$.

The following theorem is now easily proved.

THEOREM 3.6. Let $A$ be a commutative noetherian ring of $\operatorname{dim} 2$ and $X=\{M \in \max (A) \mid$ ht $M \leqslant 1\}$. Assume that $Y=X \cup \operatorname{sing}(A) \subset Z$ where $Z \subset \operatorname{spec}(A)$ is a closed set of $\operatorname{dim}<1$. Then the following are equivalent.

(1) $S K_{0}(A)=0$.

(2) All f.g. projective A modules of constant rank stably have the form free $\oplus$ rank 1.

(3) $[A / M]=0$ in $K_{0}(A)$ for all $M \in \max (A) \backslash Y$.

(4) $[A / M]=0$ in $K_{0}(A)$ for all $M \in \max (A) \backslash Z$.

Corollary 3.7. Let $A, X, Y$, and $Z$ be as in Theorem 3.6 and assume that the cancellation theorem for f.g. projection A-modules holds. Then the following are equivalent.

(1) $S K_{0}(A)=0$.

(2) All f.g. projective A-modules of constant rank have the form free $\oplus$ rank 1.

(3) $[A / M]=0$ in $K_{0}(A)$ for all $M \in \max (A) \backslash Y$.

(4) $[A / M]=0$ in $K_{0}(A)$ for all $M \in \max (A) \backslash Z$.

(5) Each $M \in \max (A) \backslash Y$ can be generated by 2 elements.

Proof. Clearly (1)-(4) are equivalent. (3) $\Rightarrow(5)$. By Lemmas 3.1 and 3.2, for each $M \in \max (Y)$ there is a SES $0 \rightarrow A \rightarrow P \rightarrow M \rightarrow 0$ where $P$ is a f.g. projective $A$-module of rank 2 with $\Lambda^{2}(P) \approx A$. By $(2), P \approx A \oplus \Lambda^{2}(P) \approx$ $A^{2}$, so $M$ can be generated by 2 elements.

(5) $\Rightarrow(3)$. For each $M \in \max (A) \backslash Y$ there is a SES $0 \rightarrow L \rightarrow A^{2} \rightarrow M \rightarrow 0$ 
with $L \approx \Lambda^{2}\left(A^{2}\right) \approx A$ by Lemma 3.2 again. Thus $[A / M]=[A]-[M]=[A]$ - $\left(\left[A^{2}\right]-[A]\right)=0$ in $K_{0}(A)$.

REMARK 3.8. If $A$ is an affine subring of $B=k[X, Y]$ with $A \subset B$ integral, then all maximal ideals of $A$ have ht 2 and $\operatorname{sing}(A)$ is closed of $\operatorname{dim}<1$. So by Theorem 3.6, $S K_{0}(A)=0$ iff $[A / M]=0$ for all $M \in \max (A) \backslash \operatorname{sing}(A)$.

\section{Main theorem.}

THEOREM 4.1. Let $k$ be an algebraically closed field and $A$ an affine subring of $B=k[X, Y]$ with $A \subset B$ integral and $\operatorname{sing}(A)$ finite. Then $S K_{0}(A)=0$, so all f.g. projective $A$-modules have the form free $\oplus$ rank 1 .

$k$ is algebraically closed, so by Theorem 2.4 the cancellation theorem holds for f.g. projective $A$-modules. So by Remark 3.8 and Corollary 3.7 it is enough to show that $[A / M]=0$ in $K_{0}(A)$ for all $M \in \max (A) \backslash \operatorname{sing}(A)$.

Let $X=\{P \in \operatorname{spec}(B) \mid P \cap A \in \operatorname{sing}(A)\}$; then $X \subset \max (B)$ and is finite since $A \subset B$ is integral and $\operatorname{sing}(A)$ is finite. Next we show that we can always avoid a finite number of maximal ideals of $B$. Geometrically this just says that given any finite set of points in $k^{2}$, there is a line which does not pass through any of these points.

LEMMA 4.2. Let $k$ be an algebraically closed field and $B=k[X, Y]$. Let $Z \subset \max (B)$ be finite and $M \in \max (B) \backslash Z$. Then there is $a P=(a X+b Y$ $+C) \subset M$ so that $P \not \subset N$ for any $N \in Z$.

PRoof. $k$ is algebraically closed, so we may assume that $M=(X, Y)$ and $Z=\left\{M_{1}, \ldots, M_{n}\right\}$ where each $M_{i}=\left(X-a_{i}, Y-b_{i}\right)$. There are only a finite number of ratios $b_{i} / a_{i}$, and since $k$ is infinite, there exists a $0 \neq a \in k$ so that $a \neq-b_{i} / a_{i}$ for $1 \leqslant i \leqslant n$. Let $P=(a X+Y) \subset M=(X, Y)$; clearly $P$ is not contained in any $M_{i}$.

$A \subset B$ is integral, so each $M \in \max (A) \backslash \operatorname{sing}(A)$ can be lifted to a $\bar{M} \in \max (B) \backslash X$. By Lemma 4.2 there is a $P=(a X+b Y+C) \subset \bar{M}$ which is not contained in any $N \in X$. Let $Q=P \cap A$; then $0 \neq Q \in \operatorname{spec}(A)$ is not contained in any element of $\operatorname{sing}(A)$. We also note that $Q$ is locally principal, and hence invertible (projective). For $M \in \operatorname{sing}(A), Q_{M}=A_{M}$, while for $M \notin \operatorname{sing}(A), A_{M}$ is a regular local ring and so ht 1 prime ideals are principal because $A_{M}$ is factorial. We also note that $\mathrm{hd}_{A} A / Q=1$. So we have shown

LeMmA 4.3. Let $M \in \max (A) \backslash \operatorname{sing}(A)$; then $M$ contains an invertible prime ideal which is not contained in any $N \in \operatorname{sing}(A)$.

We have $k \subset A / Q \subset k[X, Y] /(a X+b Y+c) \approx k[T]$, which is an integral extension. As $A / Q$ is a 1-dimensional affine domain over $k$ contained in $k[T]$, by Luroth's Theorem, the integral closure of $A / Q$ is 
$\overline{A / Q}=k[g(T)]$. So we may assume that $\overline{A / Q}=k[T]$.

LEMMA 4.4. Let $k$ be an algebraically closed field and $R$ an affine domain over $k$ of $\operatorname{dim} 1$. If ${ }_{R} \bar{R}$ is f.g., where $\bar{R}$ is the integral closure of $R$, then the natural map f: $G_{0}(\bar{R}) \rightarrow G_{0}(R)$ is surjective.

Proof. $f$ is well defined because ${ }_{R} \bar{R}$ is f.g. $G_{0}(R)$ is generated by $[R]$ and $[R / M]$ for $M \in \max (R)$. For $M \in \max (R)$, let $\bar{M} \in \max (\bar{R})$ lie over $M$. Then $R / M \hookrightarrow \bar{R} / \bar{M}$ is an $R$-isomorphism because $k$ is algebraically closed. Since $\operatorname{rank}_{R} \bar{R}=1$, also $[R] \in \operatorname{im} f$. So $f$ is surjective.

LEMMA 4.5. Let $R$ be a domain with $G_{0}(R) \approx \mathrm{Z}$; then $[R / I]=0$ in $G_{0}(R)$ for all $0 \neq I \in \operatorname{spec}(R)$.

Proof. Since $R$ is a domain, we have the SES $0 \rightarrow$ ker $r k \rightarrow G_{0}(R) \rightarrow^{r k} \mathbf{Z} \rightarrow$ 0 . $G_{0}(R) \approx \mathrm{Z}$, so ker $r k=0$. But clearly each $R / I$ has $\operatorname{rank} 0$, so $[R / I]=0$ in $G_{0}(R)$.

In our case $R=A / Q$ and $\bar{R}=\overline{A / Q}=k[T] . G_{0}(k[T]) \approx \mathbf{Z}$ and $G_{0}(A / Q)$ $\approx \mathbf{Z} \oplus \tilde{G}_{0}(A / Q)$ since $A / Q$ is a domain. By Lemma $4.4, \tilde{G}_{0}(A / Q)=0$, so $G_{0}(A / Q)=\mathbf{Z}$ on $[A / Q]$. So for each $M \in \max (A) \backslash \operatorname{sing}(A), M$ contains an invertible prime ideal $Q$ which misses $\operatorname{sing}(A)$. So $G_{0}(A / Q)=\mathbf{Z}$ and thus $[A / M]=0$ in $G_{0}(A / Q)$ by Lemma 4.5.

If $N$ is a f.g. $A / Q$-module, then $N$ is also a f.g. $A$-module and clearly $\operatorname{supp}(N) \subset V(Q)$. Since $\operatorname{hd}_{A} A / Q=1$ and $V(Q) \cap \operatorname{sing}(A)=\varnothing$, each f.g. $A / Q$-module also has finite homological dimension when considered as an $A$-module. We thus have a natural homomorphism $\psi: G_{0}(A / Q) \rightarrow K_{0}(A)=$ $K_{0}(H(A))$ defined by $\psi([A / Q])=[A / Q]$. But $[A / M]=0$ in $G_{0}(A / Q)$ and, hence, $[A / M]=\psi([A / M])=0$ in $K_{0}(A)$ also. So we have shown that $[A / M]=0$ in $K_{0}(A)$ for all $M \in \max (A) \backslash \operatorname{sing}(A)$, and thus the proof of Theorem 4.1 is complete.

If $A$ is an affine normal domain of $\operatorname{dim} 2$, then $\operatorname{sing}(A)$ is finite; so the following corollary is immediate.

COROLLARY 4.6. Let $k$ be an algebraically closed field and $A$ an affine normal subring of $B=k[X, Y]$ with $A \subset B$ integral. Then all f.g. projective $A$-modules have the form free $\oplus$ rank 1 .

ReMARK 4.7. We note that Theorem 4.1 includes more than just the normal subrings. For example, $A=k\left[X^{2}, X^{3}, X Y, Y^{2}, Y^{3}\right]$ is a subring of $k[X, Y]$ whose only singularity is the origin. Also note that $\operatorname{Pic}(A) \neq 0$.

RemarK 4.8. We do not know any examples of rings of the type in Corollary 4.6 for which $\operatorname{Pic}(A) \neq 0$.

ReMARK 4.9. In $\$ 6$ we show that Theorem 4.1 cannot be improved to dim $\operatorname{sing}(A)<1$. In fact, $A=\mathrm{C}\left[X, Y\left(X^{2}-Y\right), Y^{2}\left(X^{2}-Y\right)\right]$ has indecomposable projective modules of rank 2 . 
We do not know if it necessary for $k$ to be algebraically closed. However, we do have the following partial result.

Proposition 4.10. Let $A$ be an affine subring of $B=k[X, Y]$ with $A \subset B$ integral and $\operatorname{sing}(A)$ finite. Then $S K_{0}(A)$ is torsion.

Proof. Let $k^{\prime}$ be a finite algebraic extension of $k$ with $\left[k^{\prime}: k\right]=n<\infty$. Then $K_{0}(A) \stackrel{f}{\rightarrow} K_{0}\left(k^{\prime} \otimes_{k} A\right) \rightarrow K_{0}(A)$ is just multiplication by $n$, so ker $f$ is $n$-torsion. The algebraic closure $\bar{k}$ of $k$ is the direct limit of finite algebraic extensions of $k$, say $\bar{k}=$ proj $\lim k^{\prime}$. So

$$
\bar{A}=\bar{k} \otimes_{k} A=\left(\text { proj } \lim k^{\prime}\right) \otimes_{k} A \approx \text { proj } \lim \left(k^{\prime} \otimes_{k} A\right),
$$

and thus $K_{0}(\bar{A})=\operatorname{proj} \lim K_{0}\left(k^{\prime} \otimes_{k} A\right)$. Hence $L=\operatorname{ker}\left(K_{0}(A) \rightarrow K_{0}(\bar{A})\right)$ is also torsion. But $S K_{0}(\bar{A})=0$ by Theorem 4.1,so $S K_{0}(A) \subset L$ and, hence, is torsion.

REMARK 4.11. We are also not sure if Theorem 4.1 is valid without assuming $A \subset B$ integral. For example, let $A=\mathrm{C} X\left(X^{2}+Y^{3}\right)^{3}, Y\left(X^{2}+\right.$ $\left.\left.Y^{3}\right)^{2}, X^{2}+Y^{3}\right] \subset \mathrm{C}[X, Y]$. Then $A \approx \mathrm{C}[X, Y, Z] /\left(Z^{2}-\left(X^{3}+Y^{7}\right)\right)$, so $A$ is factorial [12, p. 31]. Thus $A$ is normal and $\operatorname{Pic}(A)=0$. Clearly $\mathrm{C}(X, Y)$ is the quotient field of $A$, but $A \subset \mathrm{C}[X, Y]$ is not integral because $A$ is normal. It is not known if all f.g. projective $A$-modules are free.

One special case when $\operatorname{Pic}(A)=0$ is when $A$ is graded.

LEMMA 4.12 [7]. Let $A=A_{0} \oplus A_{1} \oplus \ldots$ be an affine normal domain with $A_{0}$ a field; then $\operatorname{Pic}(A)=0$.

As an immediate corollary of Lemma 4.12 and Corollary 4.6, we have

COROLlaRY 4.13. Let $k$ be an algebraically closed field and $A$ an affine normal graded subring of $B=k[X, Y]$ with $A \subset B$ integral; then all f.g. projective $A$-modules are free.

5. Rings of invariants. A special case of the rings of Corollary 4.6 are rings of invariants of $k[X, Y]$. Let $A$ be a ring and $G$ a subgroup of $\operatorname{Aut}(A)$; then $A^{G}=\{a \in A \mid \theta(a)=a \forall \theta \in G\}$ is a subring of $A$. Let $B=k[X, Y]$ and $G \subset \operatorname{Aut}_{k}(B)$ be a finite subgroup; then $A=B^{G}$ is an affine normal subring of $B$ with $A \subset B$ integral. Proposition 5.1 thus follows from Corollaries 4.6 and 4.13.

Proposition 5.1. Let $k$ be an algebraically closed field and $G \subset$ $\operatorname{Aut}_{k}(k[X, Y])$ a finite subgroup. Then all f.g. projective $A=k[X, Y]^{G}$ modules have the form free $\oplus$ rank 1 . If, in addition, each $\theta \in G$ is a graded automorphism, then all f.g. projective A-modules are free.

Instead of considering rings of invariants, we can also consider the kernel 
of a group of derivations. Let $k$ be a field of char $k=p \neq 0$ and $G$ a finite group of $k$-derivations of $B=k[X, Y]$. Then $k[X, Y]^{p} \subset \operatorname{ker} D$ for each $D \in G ; A=G_{k}[X, Y]=\{f \in k[X, Y] \mid D(f)=0 \forall D \in G\}$ is a subring of $B$ with $k[X, Y]^{p} \subset{ }^{G} B \subset B$, and hence $A \subset B$ is integral. If all $D \in G$ are graded, then $A={ }^{G} B$ will also be graded. So we have the following proposition.

Proposition 5.2. Let $k$ be an algebraically closed field with char $k=p \neq 0$ and $G$ a finite group of $k$-derivations of $B=k[X, Y]$. Then all f.g. projective $A={ }^{G} B$-modules have the form free $\oplus$ rank 1 . Moreover, if all $D \in G$ are graded, then all f.g. projective $A$-modules are free.

Again, we do not know if it is necessary for $k$ to be algebraically closed. However, if $A$ is generated by monomials over $k$, then by Theorem 1.1, all f.g. projective modules are free, regardless of $k$.

6. Constructing indecomposable projective modules. For arbitrary subrings $A$ of $B=k[X, Y]$ not all f.g. projective $A$-modules have the form free $\oplus$ rank 1. In fact, this depends on the field $k$. We give several examples of subrings $A$ of $B$ with $A \subset B$ integral, but $S K_{0}(A) \neq 0$.

EXAMPLE 6.1. Let $A=k\left[X, Y\left(X^{2}-Y\right), Y^{2}\left(X^{2}-Y\right)\right] \subset B=k[X, Y]$. $A \subset B$ is integral, and $B$ is the integral closure of $A$. We note that $I=Y\left(X^{2}\right.$ $-Y) B$ is contained in the conductor of $B / A$. We then have the following cartesian square.

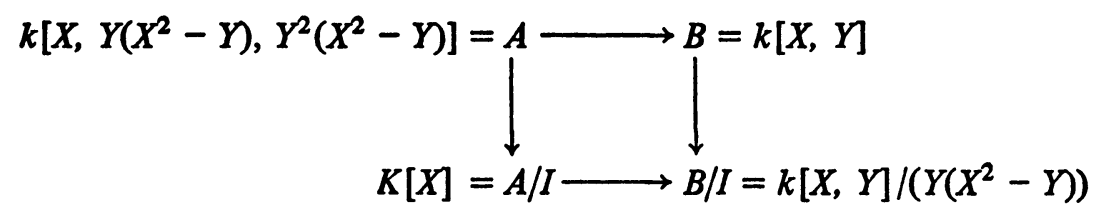

By Theorem 2.2, $\operatorname{Pic}(A)=0$ and $S K_{0}(A) \approx S K_{1}(B / I)$ by Lemma 2.3. Using the Mayer-Vietoris $K$-theory exact sequence for $K_{2}[6]$, it is easy to see that $S K_{1}(B / I) \approx K_{2}\left(k[T] /\left(T^{2}\right)\right) / K_{2}(k)$. If $k$ is a field of char $k \neq 2$ or a perfect field, then $K_{2}\left(k[T] /\left(T^{2}\right)\right) \approx K_{2}(k) \oplus \Omega_{\mathrm{Z}}^{1}$, so $S K_{0}(A) \approx \Omega_{\mathrm{Z}}^{1}[16]$.

If $k$ is a finite field, then $\Omega_{\mathrm{z}}^{1}=0$. However, by [15, p. 45], $\operatorname{sr}(A)<2$ when $k$ is a finite field, so all f.g. projective $A$-modules are free. But if $k=\mathrm{C}$, then $\Omega_{\mathbf{C} / \mathbf{Z}}^{1} \neq 0$. Thus $\operatorname{Pic}(A)=0$ while $S K_{0}(A) \neq 0$, so there exist indecomposable projective $A$-modules of rank 2. For let $0 \neq[P]-\left[A^{2}\right] \in S K_{0}(A)$. If $P$ were decomposable, then necessarily $P \approx A^{2}$ since $\operatorname{Pic}(A)=0$. Note that $P$ is not even stably decomposable. This ring was used by Pedrini [11] as an example of a ring for which $N K_{0}(A) \neq 0$, but $\operatorname{Pic}(A)=\operatorname{Pic}(A[T])=0$.

REMARK 6.2. We note that 


$$
\begin{aligned}
A & =k\left[X, Y\left(X^{2}-Y\right), Y^{2}\left(X^{2}-Y\right)\right] \\
& \approx k[U, V, W] /\left(V^{3}+W^{2}-U^{2} V W\right) .
\end{aligned}
$$

Thus $A$ is graded if we assign weights of 1,4 , and 6 to $U, V$, and $W$, respectively. If $k=\mathrm{C}$, then $\left\{(U-\alpha, V, W) /\left(V^{3}+W^{2}-U^{2} V W\right)\right\} \subset$ $\operatorname{sing}(A)$, so $\operatorname{dim} \operatorname{sing}(A)=1$. Thus the hypothesis of Theorem 4.1 cannot be improved to $\operatorname{dim} \operatorname{sing}(A)<1$.

REMARK 6.3. $A=\mathrm{C}\left[X, Y\left(X^{2}-Y\right), Y^{2}\left(X^{2}-Y\right)\right]$ is an example of an affine domain over an algebraically closed field which has an indecomposable projective module of rank 2 . This answers a question raised by Murthy [7].

It is not hard to give more examples. They depend upon calculating $S K_{1}(k[X, Y] /(f))$ for $f \in k[X, Y]$. For example, Theorem 1.1(1) really follows because $S K_{1}\left(k[X, Y] /\left(X^{i} Y^{j}\right)\right)=0$.

LEMMA 6.4. Let $k$ be algebraic over a finite field and $f \in k[X, Y]$; then $S K_{1}(k[X, Y] /(f))=0$.

Proof. By [15, p. 45], $\operatorname{sr}(k[X, Y])<2$. Assuming this, a proof may be found in $[4$, p. 29].

Proposition 6.5. Let $k$ be algebraic over a finite field and $A$ an affine subring of $B=k[X, Y]$ with integral closure $B$; then $S K_{0}(A)=0$. If $\operatorname{Pic}(A)=$ 0 , then all f.g. projective A-modules are free.

Proof. Since $B$ is the integral closure of $A$, the conductor $I$ of $B / A$ contains a $0 \neq f \in B$. By Lemma $6.4, S K_{1}(B / f B)=0$, so $S K_{0}(A)=0$ by Lemma 2.3. Since $\operatorname{sr}(A)<2$ all stably free $A$-modules are free. So if $\operatorname{Pic}(A)=$ 0 , all f.g. projective $A$-modules are free.

We give one final criterion for $S K_{0}(A)=0$.

Proposition 6.6. Let $A$ be an affine subring of $B=k[X, Y]$ with integral closure $B$. Let $I$ be the conductor of $B / A$. If $V(I) \subset \operatorname{spec}(B)$ is finite, then $S K_{0}(A)=0$.

Proof. If $V(I)$ is finite, then $B / I$ is semilocal, and so $S K_{1}(B / I)=0$. Thus $S K_{0}(A)=0$ by Lemma 2.3 .

We conclude this paper with two more examples like Example 6.1.

EXAMPLE 6.7. Let $A=k\left[X, Y^{2}, Y\left(X^{3}-Y^{2}\right)\right] \subset B=k[X, Y]$. Clearly $B$ is the integral closure of $A$ and $f=X^{3}-Y^{2}$ is in the conductor of $B / A$. From the cartesian square

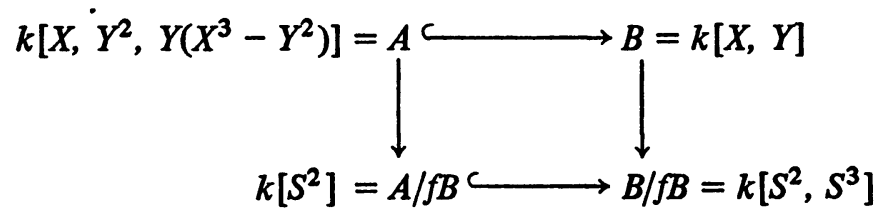


and Theorem 2.2 it is easy to calculate $\operatorname{Pic}(A)=0$ and $S K_{0}(A) \approx S K_{1}(B / f B)$ $\approx S K_{1}\left(k\left[S^{2}, S^{3}\right]\right)$.

If $k$ is algebraic over a finite field, then $S K_{1}(B / f B)=0$ by Lemma 6.4, so $S K_{0}(A)=0$. In this case all f.g. projective $A$-modules are free because $\operatorname{sr}(A) \leqslant 2$.

However, if $k$ is algebraically closed and has infinite transcendence degree over $\mathbf{Q}$, then $S K_{1}(B / f B) \approx S K_{1}\left(k\left[S^{2}, S^{3}\right]\right) \neq 0$ [8]. So in this case $A$ has indecomposable projective modules of rank 2.

EXAMPLe 6.8. Let $A=k\left[X, Y^{2}, Y\left(X^{2}+Y^{2}-1\right)\right] \subset B=k[X, Y]$. Then $B$ is the integral closure of $A$ and $f=X^{2}+Y^{2}-1$ is in the conductor of $B / A$. From the cartesian square

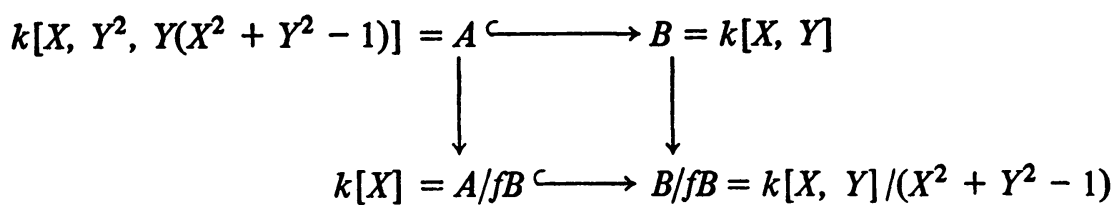

and Lemma 2.3 we see that $S K_{0}(A) \approx S K_{1}(B / f B)$. We consider three cases for $k$.

(1) $\operatorname{char} k=2$. Then $B / f B=k[X, Y] /(X+Y+1)^{2}$, so $S K_{1}(B / f B) \approx$ $S K_{1}(k[X, Y] /(X+Y+1))=0$. Thus $S K_{0}(A)=0$. By Theorem 2.2, $\operatorname{Pic}(A)$ $\neq 0$.

(2) $k=\mathbf{C}$ (or $\sqrt{-1}=i \in k$ ). Letting $U=X+i Y$ and $V=X-i Y$, then $B / f B=\mathbf{C}[X, Y] /\left(X^{2}+Y^{2}-1\right) \approx \mathbf{C}[U, V] /(U V-1) \approx \mathbf{C}[U, 1 / U]$. Thus $S K_{0}(A)=0$, while $\operatorname{Pic}(A) \approx \mathbf{Z}$. By Theorem 2.4 every f.g. projective $A$-module has the form free $\oplus$ rank 1 .

(3) $k=\mathbf{R}$. By [6, p. 128], $S K_{1}\left(\mathbf{R}[X, Y] /\left(X^{2}+Y^{2}-1\right)\right) \approx Z / 2 Z$, so $S K_{0}(A) \approx \mathbf{Z} / 2 \mathrm{Z}$. $(B / f B)^{*}=\mathbf{R}^{*}$, so $\operatorname{Pic}(A)=0$ from Theorem 2.2. Let $0 \neq$ $[P]-\left[A^{2}\right] \in S K_{0}(A)$; then $P$ is a f.g. projective $A$-module of rank 2 which is not stably free. Thus $P$ is indecomposable. However, $2\left([P]-\left[A^{2}\right]\right)=[P \oplus$ $P]-\left[A^{4}\right]=0$, so $P \oplus P \approx A^{4}$.

\section{REFERENCES}

1. D. F. Anderson, Projective modules over subrings of $k[X, Y]$, Dissertation, Univ. of Chicago, Chicago, Ill., 1976.

2. , Projective modules over subrings of $k[X, Y]$ generated by monomials (submitted).

3. H. Bass, Algebraic K-theory, Benjamin, New York, 1968.

4. M. Krusemeyer, Fundamental groups, algebraic K-theory, and a problem of Abyhyankar, Invent. Math. 19 (1973), 15-47.

5 H. Matsumura, Commutative algebra, Benjamin, New York, 1970.

6. J. Milnor, Introduction to algebraic K-theory, Princeton Univ. Press, Princeton, N. J., 1971.

7. M. P. Murthy, Vector bundles over affine surfaces birationally equivalent to a ruled surface, Ann. of Math. (2) 89 (1969), 242-253. 
8. M. P. Murthy and C. Pedrini, $K_{0}$ and $K_{1}$ of polynomial rings, Algebraic $K$-theory. II, Lecture Notes in Math., vol. 342, Springer, Berlin, 1973, pp. 109-121.

9. M. P. Murthy and R. G. Swan, Vector bundles over affine surfaces, Invent. Math. 36 (1970, 125-165.

10. M. Nagata, On the closedness of singular loci, Inst. Hautes Études Sci. Publ. No. 2, (1952), 5-12.

11. C. Pedrini, On the $K_{0}$ of certain polynomial extensions, Algebraic $K$-theory. II, Lecture Notes in Math., vol. 342, Springer, Berlin, 1973, pp. 92-108.

12. P. Samuel, Unique factorization domains (Lecture notes), Tata Institute, Bombay, 1964.

13. J. P. Serre, Sur les modules projectifs, Séminaire Dubriel-Pisot 14 (1960-61), No. 2.

14. C. S. Seshadri, Triviality of vector bundles over the affine space $K^{2}$, Proc. Nat. Acad. Sci. U.S.A. 44 (1958), 456-458.

15. R. G. Swan, Serre's problem, Conference on Commutative Algebra, Queen's Papers in Pure and Appl. Math., No. 42, Kingston, Ont., 1975.

16. W. van der Kallen, Le $K_{2}$ des nombres duaux, C. R. Acad. Sci. Paris 273 (1971), 1204-1207.

Department of Mathematics, University of Tennessee, Knoxville, Tennessee 37916 